\title{
Developmental variations of plasma gamma-glutamyltransferase fractions in humans and in laboratory mammalians
}

\author{
Vanna Fierabracci ${ }^{1}$, Maria Franzini ${ }^{2,3}$, Angelo Baggiani ${ }^{1}$, Irene Fornaciari ${ }^{2}$, Silvia Burchielli ${ }^{3}$, \\ Patrizia Urciuoli ${ }^{4}$, Roberta Lamanna ${ }^{4}$, Simone Lapi ${ }^{4}$, Francesca Nocchi ${ }^{4}$, Maria Carla Iorio ${ }^{4}$, \\ Michele Emdin ${ }^{3}$, Alfonso Pompella ${ }^{1}$, and Aldo Paolicchi ${ }^{1,3}$ \\ ${ }^{1}$ Department of Experimental Pathology, University of Pisa, Pisa, Italy, ${ }^{2}$ Scuola Superiore Sant'Anna, Pisa, Italy, \\ ${ }^{3}$ G. Monasterio Foundation CNR-Regione Toscana, Pisa, Italy, and ${ }^{4}$ Tissue and Cell Bank, Immunohaematology Unit, \\ Azienda Ospedaliera Universitaria Pisana, Pisa, Italy
}

\begin{abstract}
Plasma samples from human cord blood, and fetuses, newborns, and adults of different mammalians species were analyzed by gel-filtration chromatography, to ascertain whether gamma-glutamyltransferase (GGT) fractions reflect liver maturation. Human cord blood plasma showed higher b-, m-, and s-GGT fraction as compared to adult women. In rat and mouse fetuses and in newborns, b-GGT was the most abundant fraction. As in adult humans, in adult rats, mice, rabbits, sheep, and mini pigs, f-GGT was the most abundant fraction. GGT fractions are a common feature of all mammalian species tested. Their pattern changes seem to reflect liver postnatal maturation, function.
\end{abstract}

Keywords: Liver, gel-filtration chromatography, fetus, human umbilical cord blood, mother, mammalian

\section{Introduction}

Gamma-glutamyltransferase (GGT) is a cell-membrane enzyme that catalyses the hydrolysis of extracellular glutathione (Whitfield, 2001). Although virtually all cell types display GGT activity, and high activity is attained in several tissues, including kidney, bile ducts, choroid plexus, and testis, serum GGT is thought to derive exclusively from liver and is used as a biomarker of liver dysfunction and excessive alcohol use (Whitfield, 2001).

Serum GGT values have been positively associated with the risk of cardiovascular events (Emdin et al., 2001; Lee et al., 2006; Meisinger et al., 2006; Ruttmann et al., 2005), of hypertension (Lee et al., 2003, 2005), type II diabetes (Lee et al., 2004, 2005; Lim et al., 2007; Lippi, Targher, Guidi, 2007; Targher et al., 2007), and metabolic syndrome (Lee et al., 2007), independently of liver disease and alcohol consumption; unfortunately, the currently used laboratory GGT assays do not allow to discriminate among the different causes of GGT increase, thus reducing the clinical value and specificity of this otherwise sensitive disease biomarker.

We devised a high sensitivity method that identifies, in all healthy subjects, four GGT fractions with different molecular weight (MW), that we named b-GGT, m-GGT, s-GGT, and f-GGT, with MW corresponding to 2000, 1000,250 , and $70 \mathrm{kDa}$, respectively (Franzini et al., 2008a). Although the f-GGT is the most abundant fraction in healthy adults (Franzini et al., 2008b), we found that s-GGT shows a prominent increase in liver disease (Franzini et al., 2010a), whereas b-GGT, and to a lesser extent m-GGT and s-GGT values are associated with the occurrence of cardiovascular risk factors in healthy subjects (Franzini et al., 2010b).

No other information is available as concerns the biological meaning and the physiological determinants of plasma GGT fractions, or if they exist in laboratory

Address for Correspondence: Maria Franzini, Scuola Superiore Sant'Anna, c/o G. Monasterio Foundation CNR-Regione Toscana, Via Giuseppe Moruzzi 1, 56124 Pisa, Italy. Tel: (+39)-050-3153309. Fax: (+39)-050-3152166. E-mail: m.franzini@sssup.it; franzinimaria@gmail.com 
mammalians used to study human diseases. Since in all mammalians liver is known to be structurally and functionally immature at birth, and reaches full functional maturation with age, to start answering these questions, we decided to investigate whether high-molecular weight GGT fractions change during postnatal maturation of the liver in humans and laboratory mammalians, that are known for presenting at birth with a substantially mature (Van Eyken et al., 1988a) and a highly immature (Van Eyken, Sciot, Desmet, 1988b) liver, respectively.

\section{Animals and methods}

\section{Chemicals and stock solutions}

$\gamma$-Glutamyl-7-amido-4-methylcoumarin (gGluAMC, G7261), Glycylglycine (GlyGly, G1002) and all chemicals were purchased from Sigma-Aldrich. gGluAMC stock solution was prepared $4.5 \mathrm{mmol} / \mathrm{L}$ in ethanol $30 \% \mathrm{w} / \mathrm{w}$ containing $0.01 \mathrm{~N} \mathrm{NaOH}$ and stored at $-20^{\circ} \mathrm{C}$. This solution was diluted daily 25 -fold into $0.25 \mathrm{M}$ Tris- $\mathrm{HCl}$ buffer $\mathrm{pH} 8.5\left(25^{\circ} \mathrm{C}\right)$.

\section{Maternal and fetal blood samples}

Maternal and cord plasma-EDTA samples at delivery were obtained from the blood and umbilical cord blood bank of the University Hospital of Pisa; samples obtained after centrifugation within $4 \mathrm{~h}$ from delivery were stored at $-20^{\circ} \mathrm{C}$ and used within one month. All mothers (mean age $30.5 \pm 6$ years) gave their informed consent for the study.

\section{Animals}

Animals were housed at the animal facility of the IFCCNR; all mice, rats, and rabbits were housed in conventional cages (Tecniplast spa) under controlled 12/12 h light/dark cycle and given food and water ad libitum. Mini pigs were housed in authorized stall, under controlled 12/12 h light/dark cycle and given weighed food while water ad libitum. Sheep were housed in authorized stall under natural light/dark cycle and given food and water ad libitum

Plasma fractional GGT was studied in mice and rats all over postnatal development obtaining blood from fetuses (20th day of gestation) and newborn animals at $1,7,14,21$, and 30 days after delivery. Rodents were sacrificed administering an overdose of the anesthetic Zoletil (Virbac, srl; tiletamine and zolazepam $40 \mathrm{mg} \mathrm{kg}^{-1}$ ) by intraperitoneal puncture and blood was obtained by heart puncturing. Due to the small amount, blood samples obtained from individual fetuses and 1-day-old rats and mice were pooled; a pooled blood sample was obtained also from 7 days old mice.

In the other animals, fractional GGT was studied in adults. All animals were sedated with Zoletil and blood was obtained from auricular (mini pigs) or saphenous (rabbits) or jugular (sheep) vein.

This investigation was in accordance with the Italian law (DL-116, Jan. 27, 1992), which is conformed to the
Guide for the Care and Use of Laboratory Animals published by the U.S. National Institutes of Health (NIH Publication No. 85-23, revised 1996).

\section{GGT plasma fraction determination}

Analysis of total and fractional GGT was performed using plasma-EDTA samples $(0.02 \mathrm{~mL}$ for human samples, $0.1 \mathrm{~mL}$ for animal samples) as described previously (Franzini et al., 2008a, 2008b) using an FPLC (fast protein liquid chromatography) system (AKTA purifier, GE Healthcare Europe, Milan, Italy) equipped with a gel-filtration column (Superose 6 HR 10/300 GL, GE Healthcare Europe) and fluorescence detector (Jasco FP-2020, Jasco Europe, Lecco, Italy). Separation of fractional GGT was obtained by gel-filtration chromatography and the enzymatic activity was quantified by postcolumn injection of the fluorescent substrate for GGT, gGluAMC. Enzymatic reaction, in the presence of gGluAMC $0.030 \mathrm{mmol} / \mathrm{L}$ and glycylglycine $4.5 \mathrm{mmol} / \mathrm{L}$, proceeded for $4.5 \mathrm{~min}$ in a reaction coil (PFA, $2.6 \mathrm{~mL}$ ) kept at the $37^{\circ} \mathrm{C}$ in a water bath. The fluorescence detector operating at excitation/emission wavelengths of $380 / 440 \mathrm{~nm}$ detected the AMC signal; the intensity of the fluorescence signal was expressed in arbitrary fluorescence units (f.u.). Under this reaction conditions, area under curve is proportional to GGT activity. Total area, between 10 and $25 \mathrm{~mL}$ elution volume, and fractional GGT area was calculated by a MatLab program (Version 7 MathWorks, Inc.) to resolve overlapping peaks; the curve fitting was conducted with a nonlinear least-squares minimization algorithm using four exponentially modified Gaussian (EMG) curves. The reaction was calibrated analyzing plasma samples with known total GGT activity (standards); the slope of the calibration curve was used to convert total and fractional GGT area in activity values expressed as U/L. The sum of fractional GGT activity represents on average the $99 \%$ of total GGT activity.

\section{Statistics}

Total and fractional GGT activity levels among healthy women, puerperae, and cord blood samples were compared by 1-way ANOVA, followed by Bonferroni's Multiple Comparison Test. Total, b-, m-, s-GGT, as well as the b-GGT/s-GGT ratio were ln-transformed to reduce the distribution skewness.

\section{Results}

\section{GGT in the plasma of mothers and corresponding placental cord blood}

Mothers showed total serum GGT activity level similar to healthy adult women, while cord blood samples showed higher GGT activity in comparison with both healthy adults and mothers $(p<0.001$ in both comparisons, Table 1). In plasma samples obtained from mothers and cord blood we found the same four fractions (b-, m--, s-, f-GGT) described in the population of healthy adult women. At difference with controls, in both cases the s-GGT was the 
most abundant fraction: this peculiar GGT fraction pattern in mothers was because of significantly lower levels off-GGT as compared to healthy women $(p<0.001)$, while in cord blood this was because of significantly higher values of s-GGT $(p<0.001$; Table 1$)$.

\section{GGT fractions in rat and mouse fetus and newborn animals}

Fractional GGT profile in rat fetus at term was characterized by the abundance of b-GGT, which was the prominent fraction (Table 2). Fractional GGT pattern changed sharply $24 \mathrm{~h}$ after delivery, when b-GGT activity decreased, and f-GGT, and to a larger extent s-GGT increased (Table 2). During the following weeks, all GGT fraction activities gradually decreased, but with different rate, and at weaning most of the circulating GGT was present as f-GGT activity, like in adults (Table 2)

Also in mice, b-GGT was the most abundant fraction in fetus at term, and then decreasing sharply to become the third most abundant fraction at day 1 after birth. The pattern of decrease of individual GGT fraction in mice was substantially the same than in rats, to leave f-GGT as the only circulating fraction at 30 days of age (Table 2).

\section{GGT fractions adult sheep, rabbit, and mini pig}

Adult sheep exhibited the highest GGT activity among all species tested, mostly in the form of f-GGT (Table 3). The f-GGT was the main GGT fraction also in rabbits and mini pigs, but the remaining GGT was mostly represented by s-GGT in rabbits, and by b-GGT in mini pigs (Table 3).

\section{Discussion}

In this study, we show for the first time that GGT fractions corresponding to those found in humans (Franzini et al., $2008 \mathrm{~b}$ ) exist also in laboratory mammalians, that the high molecular weight fractions (b-GGT, m-GGT, s-GGT) are relatively abundant in earlier stages of development in humans and animals, and that the physiological decrease of serum GGT activity, that occurs after birth, is

Table 1. Total and fractional GGT values (U/L).

\begin{tabular}{|c|c|c|c|c|c|c|}
\hline & $\begin{array}{l}\text { Healthy women } \\
\quad(n=100)\end{array}$ & $\begin{array}{l}\text { Mothers } \\
(n=6)\end{array}$ & $\begin{array}{l}\text { Cord blood } \\
\quad(n=6)\end{array}$ & $\begin{array}{l}\text { Healthy women } \\
\text { versus mother }\end{array}$ & $\begin{array}{c}\text { Healthy women } \\
\text { versus cord blood }\end{array}$ & $\begin{array}{c}\text { Mother versus } \\
\text { cord blood }\end{array}$ \\
\hline Total GGT & $16.1 \pm 6.9$ & $11.6 \pm 4.8$ & $91.4 \pm 48.0$ & n.s. & $<0.001$ & $<0.001$ \\
\hline $\mathrm{b}-\mathrm{GGT}^{\mathrm{a}}$ & $1.6 \pm 1.6$ & $2.2 \pm 0.9$ & $12.7 \pm 10.1$ & n.s. & $<0.001$ & $<0.001$ \\
\hline $\mathrm{m}-\mathrm{GGT}^{\mathrm{a}}$ & $0.6 \pm 0.3$ & $0.6 \pm 0.2$ & $3.9 \pm 2.3$ & n.s. & $<0.001$ & $<0.001$ \\
\hline $\mathrm{s}-\mathrm{GGT}^{\mathrm{a}}$ & $5.0 \pm 4.0$ & $5.7 \pm 3.4$ & $62.7 \pm 35.5$ & n.s. & $<0.001$ & $<0.001$ \\
\hline f-GGT & $9.1 \pm 2.4$ & $3.1 \pm 0.9$ & $10.6 \pm 1.8$ & $<0.001$ & n.s. & $<0.001$ \\
\hline$\underline{\mathrm{b} / \mathrm{s} \text { ratio }^{\mathrm{a}}}$ & $0.3 \pm 0.2$ & $0.7 \pm 0.6$ & $0.2 \pm 0.1$ & $<0.01$ & n.s. & $<0.01$ \\
\hline
\end{tabular}

Note: Total and fractional GGT data are presented as mean \pm SD. n.s.: not significant.

${ }^{a} 1$-way ANOVA, followed by Bonferroni's Multiple Comparison Test, performed on ln-transformed data.

Table 2. Total and fractional GGT activities (U/L) in foetus and newborn animals at different time.

\begin{tabular}{|c|c|c|c|c|c|c|}
\hline$\underline{\text { Rat }}$ & $\begin{array}{c}\text { Fetus } \\
\text { Pool, } n=6\end{array}$ & $\begin{array}{c}1 \text { day } \\
\text { Pool, } n=4\end{array}$ & $\begin{array}{c}7 \text { days } \\
n=4\end{array}$ & $\begin{array}{c}14 \text { days } \\
n=5\end{array}$ & $\begin{array}{c}21 \text { days } \\
n=4\end{array}$ & $\begin{array}{c}30 \text { days } \\
n=5\end{array}$ \\
\hline Total GGT & 25.6 & 62.1 & $11.5 \pm 1.9$ & $7.7 \pm 5.0$ & $3.2 \pm 0.3$ & $4.6 \pm 0.7$ \\
\hline b-GGT & 19.6 & 11.1 & $1.5 \pm 0.3$ & $0.7 \pm 0.1$ & $0.4 \pm 0.1$ & $0.6 \pm 0.2$ \\
\hline m-GGT & n.d. & n.d. & n.d. & n.d. & n.d. & n.d. \\
\hline s-GGT & 2.0 & 31.2 & $6.2 \pm 1.5$ & $3.4 \pm 3.1$ & $0.7 \pm 0.1$ & $0.9 \pm 0.2$ \\
\hline f-GGT & 4.0 & 18.1 & $5.8 \pm 0.8$ & $3.5 \pm 1.7$ & $1.9 \pm 0.3$ & $3.0 \pm 0.4$ \\
\hline Mice & Pool, $n=10$ & Pool, $\mathrm{n}=12$ & Pool, $\mathrm{n}=5$ & $\mathrm{n}=4$ & $\mathrm{n}=3$ & $\mathrm{n}=3$ \\
\hline Total GGT & 6.5 & 2.5 & 1.7 & $1.1 \pm 1.0$ & $0.4 \pm 0.2$ & $0.3 \pm 0.04$ \\
\hline b-GGT & 3.8 & 0.5 & 0.3 & $0.3 \pm 0.3$ & n.d. & n.d. \\
\hline m-GGT & 0.2 & n.d. & n.d. & n.d. & n.d. & n.d. \\
\hline s-GGT & 1.0 & 1.0 & 0.7 & $0.3 \pm 0.1$ & $0.2 \pm 0.1$ & n.d. \\
\hline f-GGT & 1.5 & 1.0 & 0.7 & $0.6 \pm 0.4$ & $0.3 \pm 0.1$ & $0.3 \pm 0.1$ \\
\hline
\end{tabular}

Note: Data are presented as mean \pm SD. n.d.: not detectable.

Table 3. Total and fractional GGT activity (U/L) in adult mammalians.

\begin{tabular}{lccccc}
\hline & Sheep $(n=4)$ & Mini pig $(n=4)$ & Rabbit $(n=4)$ & Rat $(n=5)$ & Mouse $(n=3)$ \\
\hline Total GGT & $63.7 \pm 4.2$ & $29.4 \pm 3.7$ & $9.2 \pm 1.1$ & $0.6 \pm 0.7$ & $0.6 \pm 0.2$ \\
b-GGT & $0.9 \pm 0.5$ & $3.4 \pm 0.9$ & $0.1 \pm 0.02$ & n.d. \\
m-GGT & n.d & $0.6 \pm 0.5$ & $0.02 \pm 0.01$ & n.d. & n.d. \\
s-GGT & $4.0 \pm 0.7$ & $1.7 \pm 0.8$ & $1.6 \pm 0.3$ & $0.9 \pm 0.2$ & n.d. \\
f-GGT & $59.6 \pm 4.5$ & $24.1 \pm 3.5$ & $8.2 \pm 0.9$ & $3.0 \pm 0.4$ & $0.3 \pm 0.1$ \\
\hline
\end{tabular}

Note: Data are presented as mean \pm SD. 
due mostly to the progressive decrease of these fractions, while f-GGT remains as the most abundant circulating GGT fraction in adults of all species.

Both in rodents and humans, the GGT activity in fetal liver and blood is significantly higher than in newborns, and it decreases progressively with postnatal maturation (Mohan, Ling, Watkins, 1994). These high activities are caused by increased transcriptional regulation of GGT genes in fetal liver (el Yaaghoubi etal., 1995), which decrease after birth. Anyway, it is unlikely that the change in GGT fraction pattern is because of changes in gene expression, as only one peptide with enzyme activity is encoded by the GGT genes (Selvaraj \& Balasubramanian, 1985), whereas the several fractions found in plasma correspond to the association of the same GGT peptide with different carriers (Nemesánszky \& Lott, 1985). The present findings suggest that during liver maturation, in parallel with a decrease of GGT expression, a change occurs in the processes leading to the formation of GGT fractions. Thus the modification of GGT fraction pattern in postnatal liver maturation likely reflects the functional and structural maturation of the liver rather than changes in gene expression.

In mammalians, the liver is known for reaching a full structural and functional maturation only after birth, but with differences among species: in humans, the development of the intrahepatic bile ducts starts at the 9th week of gestation, when ductal cells appear around large portal veins; tubular structures are formed, and gradually get incorporated in the connective tissue surrounding the portal vein, resulting in the appearance of individualized bile ducts (Van Eyken et al., 1988a). In rodents, because of the far shorter gestation ( 3 weeks instead of 40 weeks), even at 10 days of age, the bile duct system is still immature, and around the smaller portal vein branches, rings of cells are still undergoing transformation into bile ducttype cells (Van Eyken, Sciot, Desmet, 1988b).

In view of this, the findings that (i) rat fetus displays mostly b-GGT, whereas human fetus shows a more complex pattern, including fractions with lesser molecular weight, (ii) postnatal growth is accompanied by a progressive decrease of GGT fractions with higher molecular weight, and (iii) f-GGT is the dominant GGT fraction in adults of all species, suggest that the appearance of GGT fractions with smaller molecular weight reflects the progressive maturation of the liver and in particular of the biliary system; thus the alteration of the GGT fraction pattern found in human disease (Franzini et al., 2010a) is likely to be a specific consequence of the structural derangement and dysfunction of the liver rather than a simple marker of cell damage. This hypothesis is in line with findings in epithelial cell lines deriving from nonhepatic tumors overexpressing GGT, that were found to release a high molecular weight GGT fraction corresponding to b-GGT but not the other GGT fractions in the culture medium (Franzini et al., 2009a), thus showing that only functionally mature and structurally organized hepatocytes allow the formation of other GGT fractions and in particular of $\mathrm{f}-\mathrm{GGT}$.
The structural maturation of liver does not seem to be the only determinant of the GGT fraction pattern: interestingly, although mothers showed the same serum GGT values that healthy women, GGT fraction analysis revealed a significantly lower f-GGT value, and a significantly higher ratio between b-GGT and s-GGT, showing that even in the absence of changes in serum GGT activity, the GGT fraction pattern may reflect the response of liver to physiological and hormonal stimuli such as those associated to pregnancy. This may apply also to the differences in GGT fraction pattern among species, which despite showing f-GGT as the main fraction, showed largely different absolute values of individual GGT fractions and different relative distribution of their values.

Besides providing a more sensitive and informative diagnostic and prognostic marker than total GGT, GGT fractions might also allow a better understanding the pathogenesis of the diseases associated with its increase: GGT is known for participating to multiple processes in physiology and in pathology, including the generation of reactive oxygen species and free radicals and the oxidation of LDL lipoprotein (Paolicchi et al., 1999); the different biological properties of the different GGT fractions might influence their ability of contributing to the pathogenesis of human diseases. In particular, the properties of the "fetal" GGT fraction, b-GGT, appear critical for understanding the connection between liver damage, serum GGT increase, and the prognostic value of serum GGT as concerns metabolic and cardiovascular disease (Emdin et al., 2001; Lee et al., 2007). In fact, only the b-GGT was found to accumulate in atherosclerotic plaques, where it was found to colocalize with oxidized LDL lipoproteins and CD68+ foam cells, contributing to the oxidative and inflammatory events leading to plaque progression and rupture (Emdin, Pompella, Paolicchi et al., 2005; Franzini et al., 2009b; Paolicchi et al., 2004).

In conclusion, in humans and laboratory mammalians, GGT fractions pattern reflects liver function and structural maturation; studying the derangement of the liver fraction pattern associated with liver diseases might allow a better understanding of their pathogenesis as well as an additional and more specific diagnostic tool. The present study provides a basis for further studies concerning the causes and mechanisms of the GGT fraction pattern derangement in disease and for setting up experimental animal models aimed to investigate the biogenesis and function of GGT fractions.

\section{Declaration of interest}

The authors report no declarations of interest.

\section{References}

el Yaagoubi M, Visvikis A, Siest G, Wellman M. (1995). Developmentaland tissue-specific DNA methylation patterns and expression of rat gamma-glutamyltransferase. Biochem Mol Biol Int 36:257-264. 
Emdin M, Passino C, Michelassi C, Titta F, Labbate A, Donato L, Pompella A, Paolicchi A. (2001). Prognostic value of serum gamma-glutamyl transferase activity after myocardial infarction. Eur Heart j 22:1802-1807.

EmdinM,PompellaA,PaolicchiA.(2005).Gamma-glutamyltransferase, atherosclerosis, and cardiovascular disease: triggering oxidative stress within the plaque. Circulation 112:2078-2080.

Franzini M, Bramanti E, Ottaviano V, Ghiri E, Scatena F, Barsacchi R, Pompella A, Donato L, Emdin M, Paolicchi A. (2008a). A high performance gel filtration chromatography method for gammaglutamyltransferase fraction analysis. Anal Biochem 374:1-6.

Franzini M, Corti A, Fornaciari I, Balderi M, Torracca F, Lorenzini E, Baggiani A, Pompella A, Emdin M, Paolicchi A. (2009a). Cultured human cells release soluble gamma-glutamyltransferase complexes corresponding to the plasma b-GGT. Biomarkers 14:486-492.

Franzini M, Corti A, Martinelli B, Del Corso A, Emdin M, Parenti GF, Glauber M, Pompella A, Paolicchi A. (2009b). Gammaglutamyltransferase activity in human atherosclerotic plaques-biochemical similarities with the circulating enzyme. Atherosclerosis 202:119-127.

Franzini M, Fornaciari I, Siciliano G, Volpi L, Ricci G, Marchi S, Gagliardi G, Baggiani A, Torracca F, Fierabracci V, Miccoli M, Pompella A, Emdin M, Paolicchi A. (2010a). Serum gammaglutamyltransferase fractions in myotonic dystrophy type I: differences with healthy subjects and patients with liver disease. Clin Biochem 43:1246-1248.

Franzini M, Ottaviano V, Fierabracci V, Bramanti E, Zyw L, Barsacchi R, Scatena F, Boni C, Mammini C, Passino C, Pompella A, Emdin M, Paolicchi A. (2008b). Fractions of plasma gammaglutamyltransferase in healthy individuals: reference values. Clin Chim Acta 395:188-189.

Franzini M, Paolicchi A, Fornaciari I, Ottaviano V, Fierabracci V, Maltinti M, Ripoli A, Zyw L, Scatena F, Passino C, Pompella A, Emdin M. (2010b).Cardiovascular riskfactors and gamma-glutamyltransferase fractions in healthy individuals. Clin Chem Lab Med 48:713-717.

Lee DH, Jacobs DR Jr, Gross M, Kiefe CI, Roseman J, Lewis CE, Steffes M. (2003). Gamma-glutamyltransferase is a predictor of incident diabetes and hypertension: the Coronary Artery Risk Development in Young Adults (CARDIA) Study. Clin Chem 49:1358-1366.

Lee DH, Jacobs DR Jr, Gross M, Steffes M. (2005). Serum gamma-glutamyltransferase was differently associated with microalbuminuria by status of hypertension or diabetes: the Coronary Artery Risk Development in Young Adults (CARDIA) Study. Clin Chem 51:1185-1191.

Lee DH, Silventoinen K, Hu G, Jacobs DR Jr, Jousilahti P, Sundvall J, Tuomilehto J.(2006). Serumgamma-glutamyltransferase predictsnonfatal myocardial infarction and fatal coronary heart disease among 28,838 middle-aged men and women. Eur Heart j 27:2170-2176.

Lee DH, Silventoinen K, Jacobs DR Jr, Jousilahti P, Tuomileto J. (2004). gamma-Glutamyltransferase, obesity, and the risk of type 2 diabetes: observational cohort study among 20,158 middle-aged men and women. J Clin Endocrinol Metab 89:5410-5414.
Lee DS, Evans JC, Robins SJ, Wilson PW, Albano I, Fox CS, Wang TJ, Benjamin EJ, D'Agostino RB, Vasan RS. (2007). Gamma glutamyl transferase and metabolic syndrome, cardiovascular disease, and mortality risk: the Framingham Heart Study. Arterioscler Thromb Vasc Biol 27:127-133.

Lim JS, Lee DH, Park JY, Jin SH, Jacobs DR Jr. (2007). A strong interaction between serum gamma-glutamyltransferase and obesity on the risk of prevalent type 2 diabetes: results from the Third National Health and Nutrition Examination Survey. Clin Chem 53:1092-1098.

Lippi G, Targher G, Guidi GC. (2007). Relationship between gammaglutamyltransferase, fasting plasma glucose, and triglycerides in the general population. Clin Chem 53:1866-7; author reply 1869.

Meisinger C, Döring A, Schneider A, Löwel H; KORA Study Group. (2006). Serum gamma-glutamyltransferase is a predictor of incident coronary events in apparently healthy men from the general population. Atherosclerosis 189:297-302.

Mohan P, Ling SC, Watkins JB. (1994). Ontogeny of hepatobiliary secretion: role of glutathione. Hepatology 19:1504-1512.

Nemesánszky E, Lott JA. (1985). Gamma-glutamyltransferase and its isoenzymes: progress and problems. Clin Chem 31:797-803.

Paolicchi A, Emdin M, Ghliozeni E, Ciancia E, Passino C, Popoff G, Pompella A. (2004). Images in cardiovascular medicine. Human atherosclerotic plaques contain gamma-glutamyl transpeptidase enzyme activity. Circulation 109:1440.

Paolicchi A, Minotti G, Tonarelli P, Tongiani R, De Cesare D, Mezzetti A, Dominici S, Comporti M, Pompella A. (1999). Gammaglutamyl transpeptidase-dependent iron reduction and LDL oxidation-a potential mechanism in atherosclerosis. J Investig Med 47:151-160.

Ruttmann E, Brant LJ, Concin H, Diem G, Rapp K, Ulmer H; Vorarlberg Health Monitoring and Promotion Program Study Group. (2005). Gamma-glutamyltransferase as a risk factor for cardiovascular disease mortality: an epidemiological investigation in a cohort of 163,944 Austrian adults. Circulation 112:2130-2137.

Selvaraj P, Balasubramanian KA. (1985). Comparative structural and lectin-binding studies on gamma-glutamyltransferase from human adult liver, fetal liver and primary hepatoma. Eur J Biochem 153:485-490.

Targher G, Zoppini G, Lippi G, Guidi GC, Muggeo M. (2007). Effect of serum gamma-glutamyltransferase and obesity on the risk of dyslipidemia and poor glycemic control in type 2 diabetic patients: cross-sectional findings from the Verona Diabetes Study. Clin Chem 53:1867-9; author reply 1869.

Van Eyken P, Sciot R, Callea F, Van der Steen K, Moerman P, Desmet VJ. (1988a). The development of the intrahepatic bile ducts in man: a keratin-immunohistochemical study. Hepatology 8:1586-1595.

Van Eyken P, Sciot R, Desmet V. (1988b). Intrahepatic bile duct development in the rat: a cytokeratin-immunohistochemical study. Lab Invest 59:52-59.

Whitfield JB. (2001). Gamma glutamyl transferase. Crit Rev Clin Lab Sci 38:263-355. 